\title{
Optimal Upper Limits of Plateau Pressure for Patients With Acute Respiratory Distress Syndrome During the First Seven Days: A Meta-Regression Analysis
}

\author{
Hideto Yasuda ${ }^{\mathrm{a}, \mathrm{b}}$, Masamitsu Sanui ${ }^{\mathrm{a}, \mathrm{i}}$, Tetsuro Nishimura ${ }^{\mathrm{c}}$, Tetsuro Kamo ${ }^{\mathrm{d}}$, Eishu Nango ${ }^{\mathrm{e}}$, \\ Takayuki Abe ${ }^{b, f}$, Rachel Roberts ${ }^{f}$, Toru Takebayashi ${ }^{b}$, Satoru Hashimotog, \\ Alan Kawarai Lefor ${ }^{\mathrm{h}}$
}

\begin{abstract}
Background: The effects of plateau pressure during the initial days of mechanical ventilation on outcomes for patients with acute respiratory distress syndrome have not been fully examined. We conducted meta-regression analysis of plateau pressure during the first 7 days using randomized control trials to investigate the optimal upper limits of plateau pressure on different days of mechanical ventilation.

Methods: Randomized controlled trials comparing two mechanical ventilation strategies with lower and higher plateau pressures in patients with acute respiratory distress syndrome were included. Metaregression analysis was performed to determine the association of plateau pressure with mortality on days 1,3 , and 7 of mechanical ventilation.
\end{abstract}

Manuscript submitted November 8, 2020, accepted December 8, 2020

Published online January 12, 2021

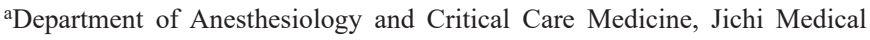
University Saitama Medical Center, 1-847 Amanuma-cho, Omiya-ku, Saitama-shi, Saitama 330-8503, Japan

${ }^{b}$ Department of Preventive Medicine and Public Health, Keio University School of Medicine, 35 Shinanomachi, Shinjuku-ku, Tokyo 160-8582, Japan 'Department of Traumatology and Critical Care Medicine, Osaka City University Graduate School of Medicine, 1-4-3 Asahi-machi, Abeno-ku, Osaka-City, Osaka 545-8585, Japan

dDepartment of Respiratory Medicine, Keio University School of Medicine, 35 Shinanomachi, Shinjuku-ku, Tokyo 160-8582, Japan

e Department of Family Medicine, JCHO Tokyo Joto Hospital, 9-13-1, Kameido, Koutou-ku, Tokyo 136-0071, Japan

fBiostatistics Unit at Clinical and Translational Research Center, Keio University Hospital, 35 Shinanomachi, Shinjuku-ku, Tokyo 160-8582, Japan gDepartment of Anesthesiology and Intensive Care, Kyoto Prefectural University of Medicine, 465 Kajii-cho, Kawaramachi-Hirokoji, Kamigyo-ku, Kyotoshi, Kyoto 602-8566, Japan

hDepartment of Surgery, Jichi Medical University, 3311-1 Yakushiji, Shimotsuke-shi, Tochigi-ken 329-0498, Japan

${ }^{\mathrm{i} C}$ Corresponding Author: Masamitsu Sanui, Department of Anesthesiology and Critical Care Medicine, Jichi Medical University Saitama Medical Center, 1-847 Amanumachou Omiya-ku, Saitama 330-8503, Japan.

Email:msanui@mac.com

doi: https://doi.org/10.14740/jocmr4390
Results: After evaluation of 2,975 citations from a comprehensive search across electronic databases, 14 studies were included in the final qualitative analysis. A total of 4,984 patients were included in the quantitative analysis. As a result of the pairwise comparison, overall short-term mortality was significantly higher for patients with plateau pressures over $32 \mathrm{~cm} \mathrm{H}_{2} \mathrm{O}$ during the first 3 days after intensive care unit (ICU) admission (day 1: relative risk (RR), 0.77; $95 \%$ confidence interval $(\mathrm{CI}), 0.66-0.89 ; \mathrm{I}^{2}=0 \%$; day 3 : RR, 0.76 ; $\left.95 \% \mathrm{CI}, 0.64-0.90 ; \mathrm{I}^{2}=0 \%\right)$, but not on day 7 (RR, 0.82; 95\% CI, $0.65-1.04 ; \mathrm{I}^{2}=16 \%$ ). Plateau pressures below $27 \mathrm{~cm} \mathrm{H}_{2} \mathrm{O}$ and 30 $\mathrm{cm} \mathrm{H}_{2} \mathrm{O}$ were not associated with an absolute risk reduction of shortterm mortality. According to univariable meta-regression analysis, mortality was significantly associated with plateau pressure on day 1 $(\beta=0.01$ (95\% CI, $0.002-0.024), P=0.02)$. On days 3 and 7 , however, no significant difference was detected. When the cutoffs were set at 27, 30 and $32 \mathrm{~cm} \mathrm{H}_{2} \mathrm{O}$ on day 1, which showed a significant difference, plateau pressure tended to be associated with increased mortality at pressures above the cut-off values, and there were no significant differences at pressures below the cut-off values, regardless of the cutoff used.

Conclusions: This study suggests that the optimal cut-off value for plateau pressure may be $27 \mathrm{~cm} \mathrm{H}_{2} \mathrm{O}$ especially during the initial period of mechanical ventilation, although this association may not continue during the latter period of mechanical ventilation.

Keywords: ARDS; Mechanical ventilation; Plateau pressure; Metaregression

\section{Introduction}

Acute respiratory distress syndrome (ARDS) is an acute, diffuse, inflammatory form of lung injury associated with a variety of etiologies. The mortality rate of the patients with ARDS is approximately $19.7-55.3 \%[1,2]$. A substantial number of ventilator strategies to reduce the plateau pressure have been proposed to minimize ventilator-induced lung injury (VILI) in patients with ARDS [1-8]. Considering the effect of these pressures on outcomes such as mortality, the effects from several perspectives must be evaluated, including at what cut-off 
values outcomes are affected, and how long these effects last.

In a recent observational study, Villar et al [7] reported that a plateau pressure above $26 \mathrm{~cm} \mathrm{H}_{2} \mathrm{O}$ is harmful, which suggests that the appropriate plateau pressure still remains to be conclusively determined. A Cochrane review [9] compared two lung ventilation strategies, protective and non-protective, not between different cut-off limits of plateau pressure. In contrast, although all randomized controlled trials (RCTs) included in a meta-analysis [10] involved a comparison of higher and lower levels for the upper limit of plateau pressures, no study compared various levels of plateau pressures. Since the relationship between the plateau pressure and mortality in ARDS may not be linear [11], it may be inappropriate to investigate the optimal upper limit of plateau pressure by a simple comparison of two different plateau pressures.

The interactions between the time course and changes in plateau pressure were not considered in a previous metaanalysis [10]. The degree of the effects of these pressures on outcomes such as mortality may be different on day 1 , day 3 , and day 7, and it is not clear how long these pressures need to be strictly controlled. However, most studies have evaluated the pressures on day 1 or the average of day 1 and day 3, so the effect of the pressure after several days, such as on day 3 and day 7, on outcomes has not been evaluated. Therefore, to investigate the optimal upper limits of plateau pressure over the course of ARDS, a simple meta-analysis of RCTs may be insufficient. Stratification based on the upper limits of plateau pressures, and by the period of ventilation along with a metaregression analysis, may be more meaningful.

The objective of this systematic review was to evaluate the optimal limits of plateau pressure with respect to mortality on days 1,3 , and 7 , by univariable meta-regression analysis, with or without determining the cut-off values for these pressures. In this analysis, the mean and standard deviation of the pressures for each arm of the RCTs were independent variables and mortality was the dependent variable.

\section{Materials and Methods}

This systematic review was designed on the basis of the Preferred Reporting Items for Systematic review and MetaAnalyses (PRISMA) statements [12]. This protocol is registered with the PROSPERO prospective register of systematic reviewers (CRD42016041924) and has been published [13]. Since this study is a systematic review and meta-analysis, the Institutional Review Board approval was not required. This study was conducted in compliance with the ethical standards of the responsible institution on human subjects as well as with the Helsinki Declaration.

\section{Study eligibility}

Type of studies

This analysis included only published RCTs that were either full-scale or pilot studies.

\section{Type of participants}

This study included adults (age 18 years or older) with ARDS or acute lung injury from any cause, as defined by the Berlin definition [14] or the North-American-European Consensus Conference on ARDS, undergoing mechanical ventilation (MV) [15].

\section{Types of interventions and comparators}

We included RCTs that compared two different MV strategies with lower and higher plateau pressures in patients with ARDS and acute lung injury. The values of the plateau pressures were directly extracted from each paper. High-frequency oscillation ventilation (HFOV) was excluded due to the lack of information on the plateau pressure.

\section{Type of outcomes}

The following outcome measures were evaluated: the primary outcome was short-term mortality (1: at the end of the followup period for each trial, 2: at day 28, and 3: at discharge from the hospital).

\section{Information sources}

We searched MEDLINE via the NCBI Entrez system, the Cochrane Central Register of Controlled Trials (CENTRAL), EMBASE, and Ichushi, a database of papers in the Japanese language.

\section{Search strategy}

We used the search terms "mechanical ventilation" AND "ARDS," "adult respiratory distress syndrome," "ALI" or "acute lung injury" AND "tidal volume," "pressure limited" or "volume limited." Searches were performed in May 2019. The detailed search strategy and the dates on which the searches were performed are shown here (Supplementary Material 1, www.jocmr.org).

\section{Study records and data management}

The literature search results from each database were extracted into Microsoft (Redmond WA USA) Excel files, and duplicates were removed by sorting the results alphabetically based on author. All full text files were managed with EndNote (X7) bibliographic software (Thompson Reuters, Philadelphia, Pennsylvania, USA). A meta-analysis was conducted with Review Manager (RevMan) software V.5.3.5.

\section{Selection process}

Two of the three physicians involved in the study $(\mathrm{HY}, \mathrm{TN}$, 
TK) screened each title and abstract of relevant studies during the first screening and the full text during the second screening. Disagreements were resolved by discussion with the physician who did not screen the study in question.

\section{Data collection process}

After extracting meta-analyses during the second screening, data were extracted from each study by three reviewers (HY, TN, TK) using two tools: 1) the Cochrane Data Collection Form (RCTs only) [16] and 2) Review Manager (RevMan) software V.5.3.5 [10].

\section{Risk of bias in individual studies}

The risk of bias in each included study was evaluated with the Cochrane Risk of Bias Assessment tool [17, 18] with respect to the eight domains. Each bias was graded as "low-risk," "unclear-risk," or "high-risk." Two of the three reviewers (HY, TN, TK) separately graded the bias of each study, and any disagreement was resolved by the decision of the remaining reviewer.

\section{Data synthesis}

Forest plots were used for the meta-analysis, and the effect size expressed as relative risk (RR) with $95 \%$ confidence intervals (CI) for categorical data and as weighted mean differences with 95\% CI for continuous data. Outcome measures were pooled using a random effect model to account for study-specific effects in measures. For all analyses, a two-sided $\mathrm{P}$ value $<0.05$ was considered significant. If a study was missing data, we attempted to contact the authors of the study for additional data. If a reply from the authors was not obtained, we classified it as missing data.

On days 1, 3, and 7 of mechanical ventilation, univariable meta-regression analysis was performed to evaluate the association of plateau pressure with mortality, with or without determining the cut-off values for the pressures $\left(27 \mathrm{~cm} \mathrm{H}_{2} \mathrm{O}, 30 \mathrm{~cm}\right.$ $\mathrm{H}_{2} \mathrm{O}$ and $32 \mathrm{~cm} \mathrm{H}_{2} \mathrm{O}$ ). These cut-off values were determined by the authors of this review according to the data distribution included in this review. Since the range of plateau pressures included in this review was approximately from $25 \mathrm{~cm} \mathrm{H}_{2} \mathrm{O}$ to $35 \mathrm{~cm} \mathrm{H} \mathrm{H}_{2} \mathrm{O}$, the cut-off values were set to these three plateau pressures. The mean and standard deviation of plateau pressure of each arm of the RCTs included in the analysis were the independent variables and mortality was the dependent variable. Data expressed as the median and interquartile range were converted to the mean and standard deviation values. Metaregression analysis was performed with $\mathrm{R}$ version 3.3.2.

\section{Assessment of heterogeneity}

The heterogeneity between trials for each outcome was assessed with an $\mathrm{I}^{2}$ statistic for quantifying inconsistency (RevMan). $\mathrm{I}^{2}$ values of $<25 \%, 25-50 \%$ and $>50 \%$ represented small, medium, and large amounts of heterogeneity, respectively [19].

\section{Assessment of reporting bias}

A funnel plot was planned to be used to investigate the possibility of publication bias if $\geq 10$ studies were available (RevMan) [20].

\section{Subgroup and sensitivity analyses}

Subgroup analysis was conducted based on factors that may cause heterogeneity, i.e., the cut-off values of plateau pressure. Subgroup analysis was also performed for the different time points (day 1, day 3, and day 7) that plateau pressure was measured while the patient was undergoing MV.

For sensitivity analysis, we first excluded all studies assessed as having a high risk of bias, i.e., when the quality of evidence was assessed as "low" or "very low." The remaining studies were used for sensitivity analysis.

\section{Results}

\section{Study selection}

A comprehensive search across electronic databases yielded a total of 2,975 citations (Fig. 1). After evaluation of the 2,975 citations, 99 references were evaluated in detail, and 28 were included in the qualitative synthesis. After excluding studies without detailed descriptions of plateau pressure, 14 studies $[1-4,8,21-29]$ were included in the final qualitative analysis (Fig. 1).

\section{Study characteristics}

Trial protocols for each study eventually included in this meta-analysis are summarized in Table 1 [1-4, 8, 21-29]. The total number of patients included in the quantitative analysis was 4,984. The types of interventions and comparisons varied among studies. Studies used different ventilator modes, such as volume-controlled ventilation, pressure-controlled ventilation, or airway pressure-release ventilation. The number of trials designed to compare these strategies was five for tidal volume, one for adaptive support ventilation, two for recruitment maneuver, one for airway pressure-release ventilation, five for pressurecontrolled ventilation, and four for positive end-expiratory pressure (PEEP) strategy. The risk of bias in the included studies is shown here (Supplementary Material 2, www.jocmr.org).

\section{Results of individual studies}

Table 2 [1-4, 8, 21-29] summarizes the patient characteristics and changes in respiratory variables (tidal volume, plateau 


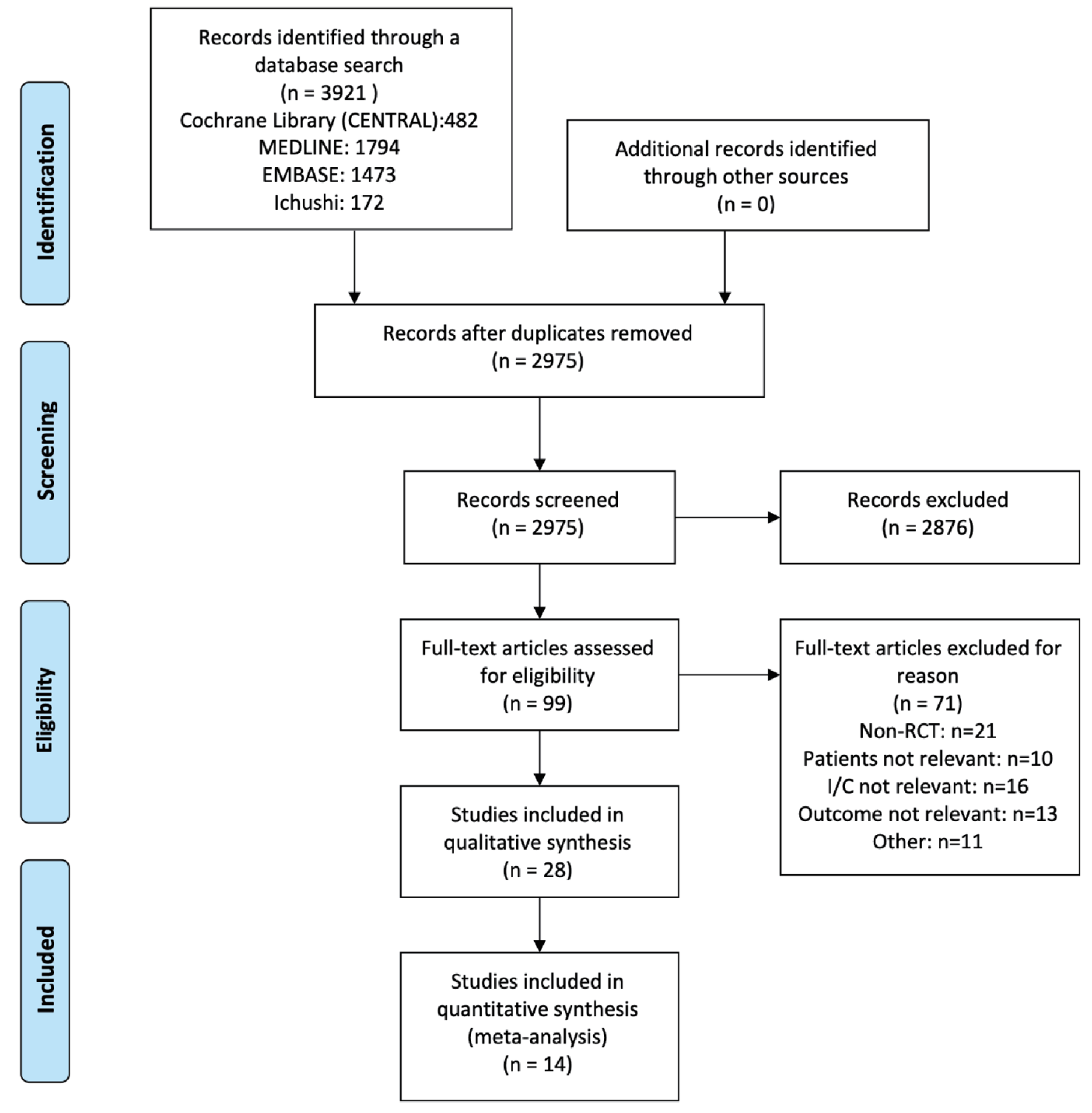

Figure 1. Flow diagram (search, inclusion, and exclusion).

pressure, and PEEP) over the course of each trial during the first 7 days after randomization. The reported daily values for tidal volume, plateau pressure, and PEEP from day 1 to day 7 differed among the studies and ranged from 5.6 to $11.8 \mathrm{~mL} / \mathrm{kg} /$ predicted body weight for tidal volume, from 18.6 to $37.0 \mathrm{~cm}$ $\mathrm{H}_{2} \mathrm{O}$ for plateau pressure and from 5.6 to 16.4 for PEEP. Based on these results, we decided to use parameters on days 1, 3, and 7 for the meta-analysis and meta-regression in this study to include as many studies as possible.

\section{Synthesis of results}

For studies with no description or an inadequate description of plateau pressure, which was essential for this meta-analysis, we contacted the authors to obtain the necessary data. Two studies [21, 24] for which the authors submitted their study data were included in this meta-analysis, but the other studies for which no response was obtained from the authors were excluded. For the assessment of reporting bias, we did not test for funnel plot asymmetry as the number of studies included in each outcome was less than 10 .

\section{Short-term mortality (main outcome and sensitivity analy-} sis)

Overall short-term mortality was significantly higher for patients with plateau pressures over $32 \mathrm{~cm} \mathrm{H}_{2} \mathrm{O}$ during the first 3 days after ICU admission (day 1: RR, 0.77; 95\% CI, 0.66 $0.89 ; \mathrm{I}^{2}=0 \%$; day $3: \mathrm{RR}, 0.76 ; 95 \% \mathrm{CI}, 0.64-0.90 ; \mathrm{I}^{2}=0 \%$; day 7: RR, 0.82; 95\% CI, $0.65-1.04 ; \mathrm{I}^{2}=16 \%$ ), but plateau 


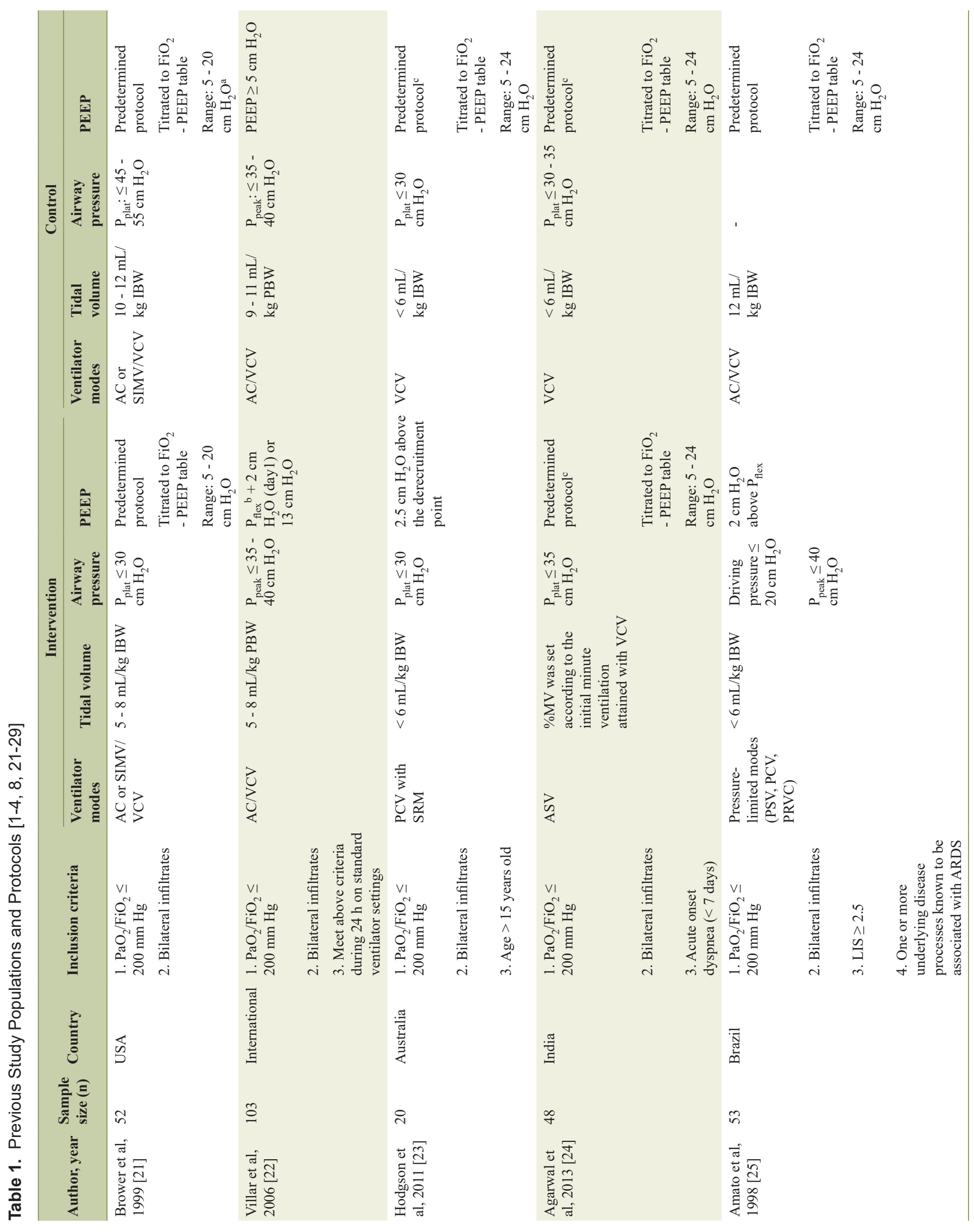




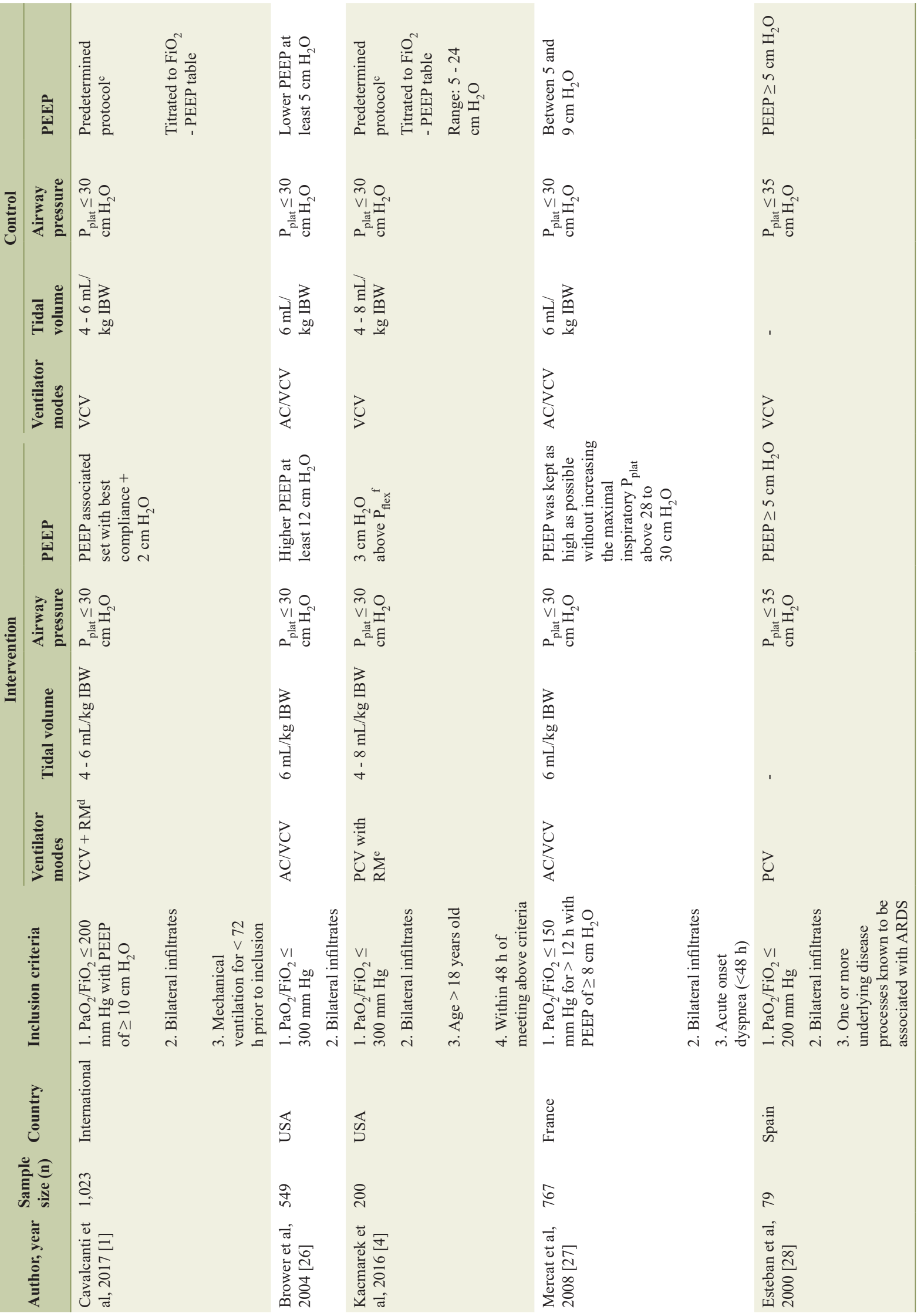




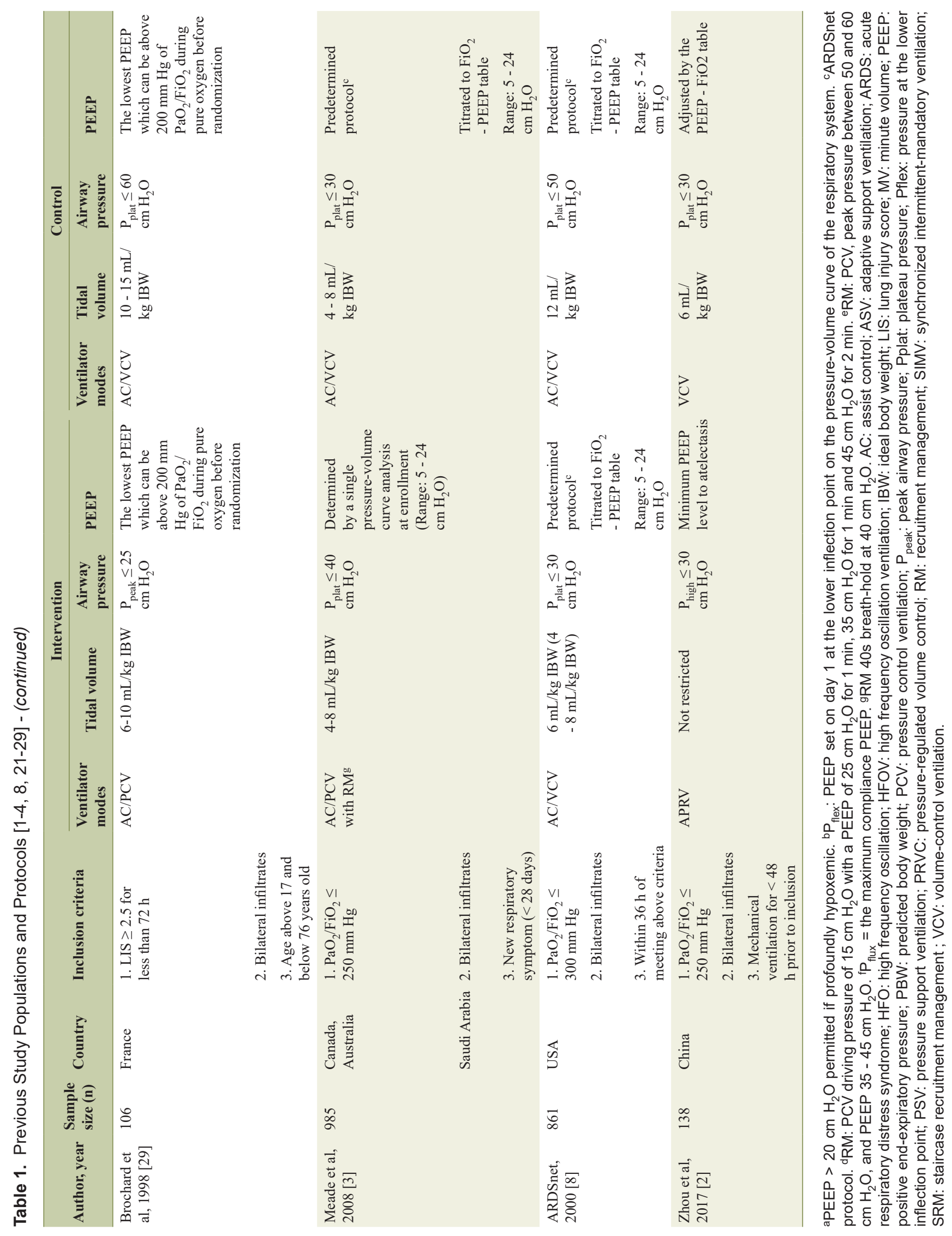



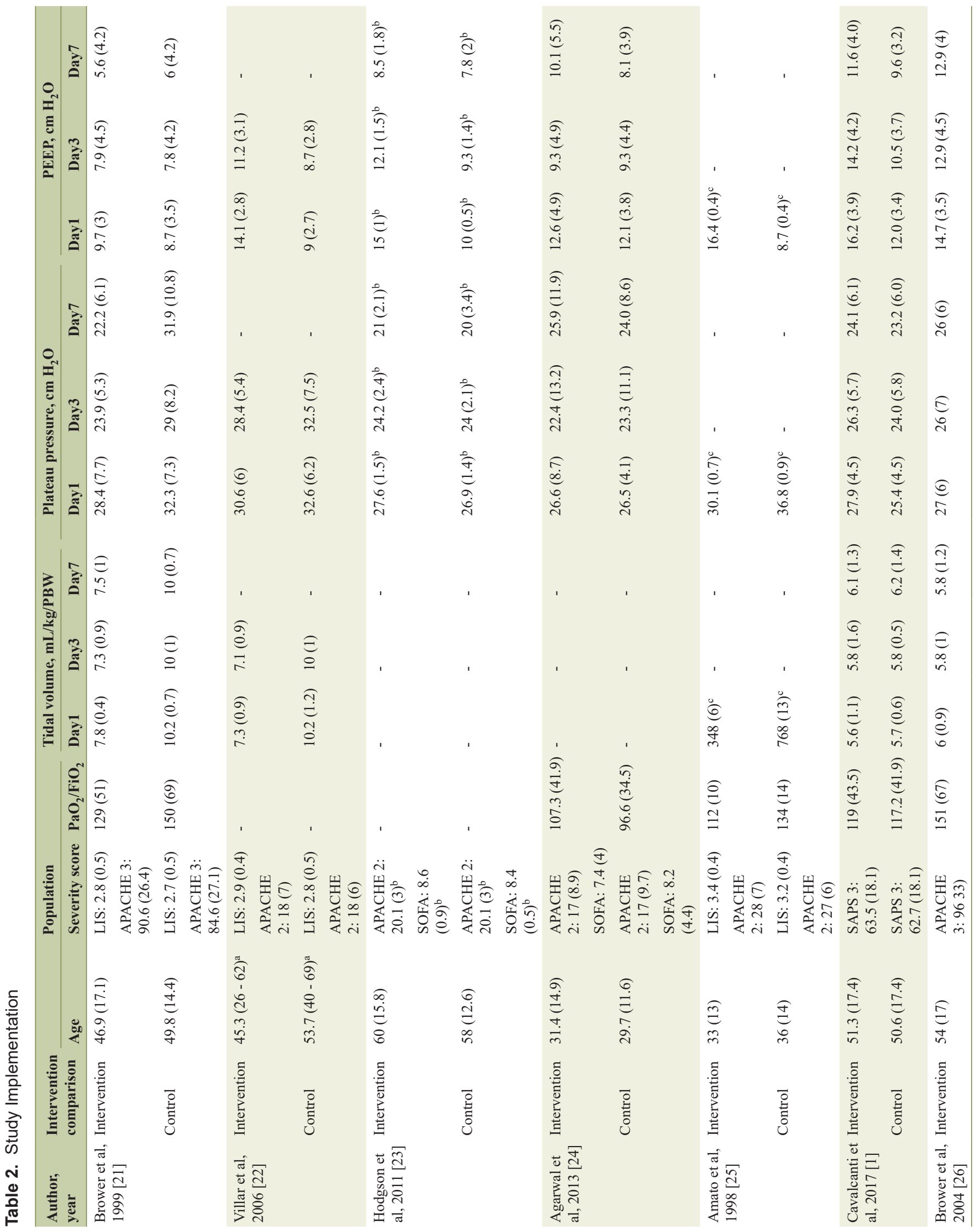


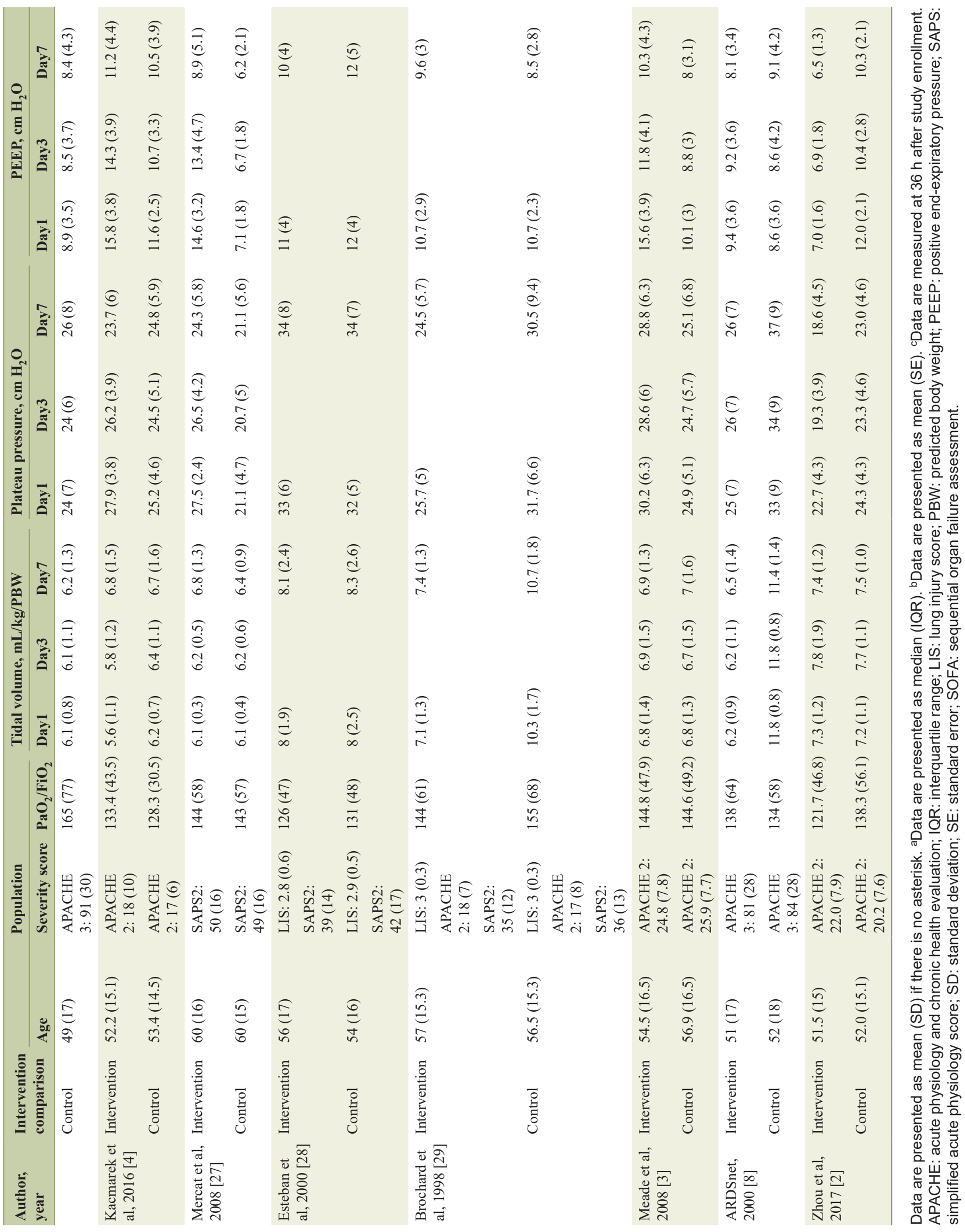


Table 3. Effect Size of Short-Term Mortality According to Plateau Pressure on Day 1, 3, and Day7 at Selected Cut-Off Values

\begin{tabular}{|c|c|c|c|c|c|c|c|c|}
\hline & $\begin{array}{l}\text { Total events } \\
\text { (below cutoff) }\end{array}$ & $\begin{array}{l}\text { Total } \\
\text { (below cutoff) }\end{array}$ & $\begin{array}{l}\text { Total events } \\
\text { (over cutoff) }\end{array}$ & $\begin{array}{l}\text { Total } \\
\text { (over cutoff) }\end{array}$ & $\mathbf{R R}$ & $95 \% \mathrm{CI}$ & Pvalue & $\mathbf{I}^{2}$ \\
\hline \multicolumn{9}{|l|}{ Day 1} \\
\hline $27 \mathrm{~cm} \mathrm{H}_{2} \mathrm{O}$ & 805 & 2,003 & 813 & 1,957 & 0.99 & 0.86 to 1.13 & 0.88 & $59 \%$ \\
\hline $30 \mathrm{~cm} \mathrm{H}_{2} \mathrm{O}$ & 379 & 1,024 & 378 & 988 & 1.00 & 0.78 to 1.28 & 0.99 & $69 \%$ \\
\hline $32 \mathrm{~cm} \mathrm{H}_{2} \mathrm{O}$ & 177 & 540 & 225 & 529 & 0.77 & 0.66 to 0.89 & 0.0007 & $0 \%$ \\
\hline $30 \mathrm{~cm} \mathrm{H}_{2} \mathrm{O}$ & 151 & 485 & 196 & 479 & 0.76 & 0.64 to 0.90 & 0.002 & $0 \%$ \\
\hline $32 \mathrm{~cm} \mathrm{H}_{2} \mathrm{O}$ & 151 & 485 & 196 & 479 & 0.76 & 0.64 to 0.90 & 0.002 & $0 \%$ \\
\hline \multicolumn{9}{|l|}{ Day 7} \\
\hline $27 \mathrm{~cm} \mathrm{H}_{2} \mathrm{O}$ & 379 & 1,024 & 378 & 988 & 1.00 & 0.78 to 1.28 & 0.99 & $69 \%$ \\
\hline
\end{tabular}

Cl: confidential interval; RR: risk ratio.

pressures below $27 \mathrm{~cm} \mathrm{H}_{2} \mathrm{O}$ and $30 \mathrm{~cm} \mathrm{H}_{2} \mathrm{O}$ were not associated with an absolute risk reduction of short-term mortality (Table 3, Supplementary Materials 3-5, www.jocmr.org).

\section{Meta-regression analysis of short-term mortality}

Since the results appeared to differ depending on the cut-off values for the plateau pressure, the mortality at the plateau pressure value was evaluated by meta-regression analysis. On day 1 , mortality tended to increase with an increase in pressure, with significant differences observed for the plateau pressure. However, on days 3 and 7, the trends showed no significant difference (Fig. 2). Since significant differences were observed for the plateau pressure on day $1(\beta=0.01$ (95\% CI, 0.002 $0.024), P=0.02$ ), plateau pressure cut-off values of 27,30 , and $32 \mathrm{~cm} \mathrm{H}_{2} \mathrm{O}$ were assessed to determine the optimal cut-off values, and regression analysis was performed. Plateau pressure tended to be associated with increased mortality at pressures above the cut-off values, especially above $27 \mathrm{~cm} \mathrm{H}_{2} \mathrm{O}$, and there were no significant differences at pressures below the cut-off values, regardless of the cutoff that was set (Fig. 3). This indicates that when the plateau pressure was below any of the three cut-off values, the mortality rate did not increase as the plateau pressure increased, but plateau pressures above 27 $\mathrm{cm} \mathrm{H}_{2} \mathrm{O}$ might affect the mortality rate. As a result, the cut-off values above $27 \mathrm{~cm} \mathrm{H}_{2} \mathrm{O}$ may affect the mortality rate. Multivariable meta-regression analysis was not performed because there was no association between age and: acute physiology and chronic health evaluation (APACHE) 2 scores and mortality rate using meta-regression analysis (Fig. 4).

\section{Quality of evidence and sensitivity analysis}

The quality of evidence for short-term mortality was evaluated at each pressure and each cut-off value (data not shown). Sensitivity analysis was not conducted because the quality of evidence of none of the included studies was assessed as "low" or "very low."

\section{Discussion}

\section{Summary of results of this review (or principal findings)}

This systematic review, using a meta-regression analysis of 14 RCTs, suggests that the plateau pressure is associated with shortterm mortality, and that this association may continue up to the first 3 days, not 1 week, after ICU admission. Optimal cut-off values that can be suggested from the results of this meta-analysis are $27 \mathrm{~cm} \mathrm{H}_{2} \mathrm{O}$ of plateau pressure from days 1 through 3 . To the best of our knowledge, this is the first systematic review to compare the effects of plateau pressure, not only on the day of ICU admission, but also several days after admission.

\section{Summary of the evidence (possible mechanisms and differ- ences from previous studies)}

Many recent meta-analyses [30-33], as well as the paper by Amato et al [34], evaluated pressures (plateau and driving pressures) on day 1 or the average pressures for a few days after starting MV. In these studies, the cut-off values for plateau pressures that influenced an improvement in the prognosis were approximately $30 \mathrm{~cm} \mathrm{H_{2 }} \mathrm{O}$ and $15 \mathrm{~cm} \mathrm{H}_{2} \mathrm{O}$. On day 1, the plateau pressure identified in the present meta-analysis was similar to the results of previous studies $[9,34,35]$. However, no previous study compared the pressures up to days 3 and 7 with prognosis, and the influence of the pressures after initiation of MV on the prognosis was unclear. The results of this review show that the influence of the pressure on mortality 

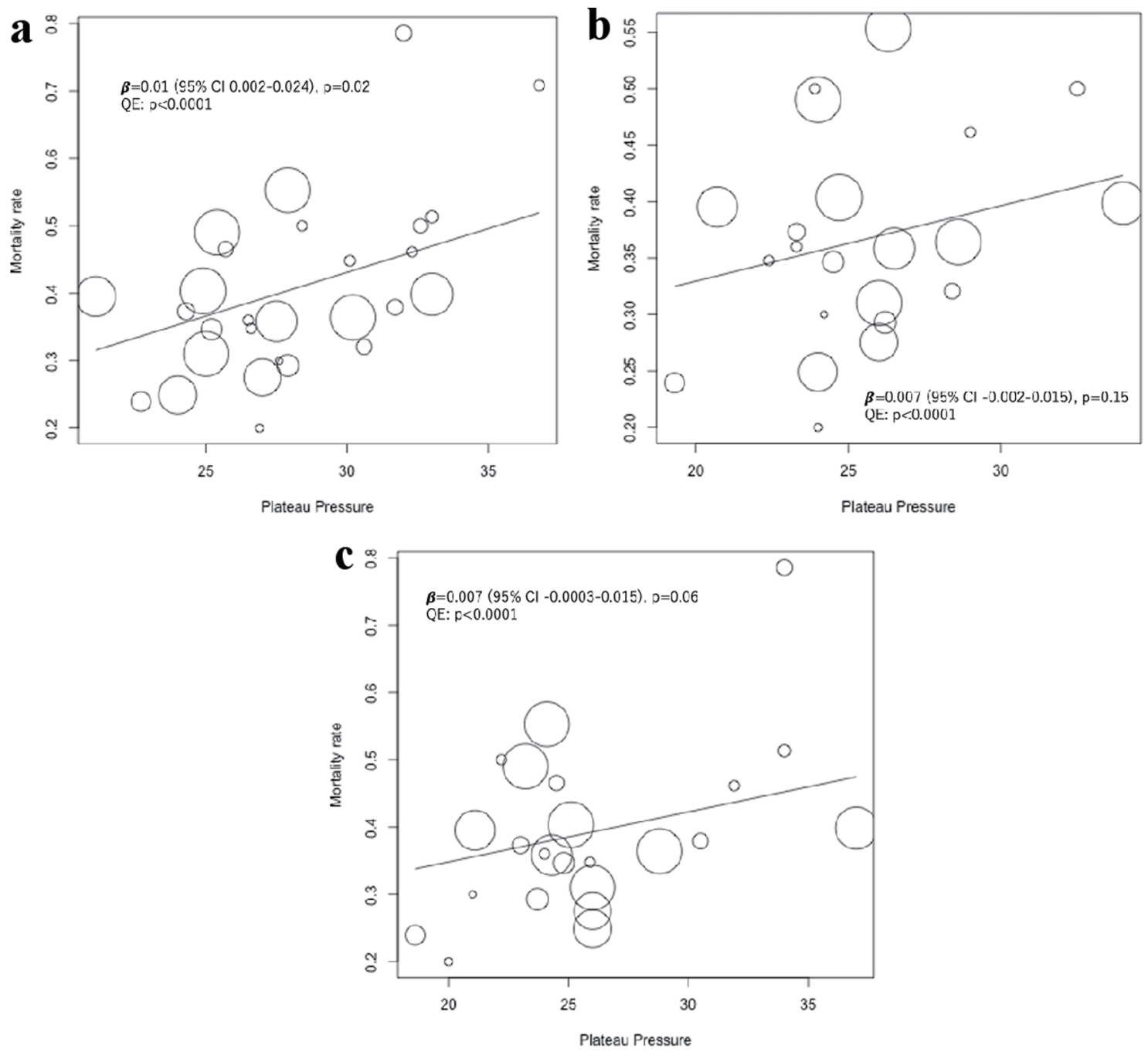

Figure 2. Univariable meta-regression analysis of the effect of plateau pressure on short-term mortality on days 1,3 and 7 . The position of each circle represents the mortality rate ( $y$-axis) and plateau pressure (x-axis) of each study arm, and circle size is proportional to sample size. (a) Meta-regression analysis of the effect of plateau pressure on day 1 on short-term mortality, including 14 studies and 28 study arms. Mean (SD) plateau pressure was 28.1 (3.7) $\mathrm{cm} \mathrm{H}_{2} \mathrm{O}$. (b) Meta-regression analysis of the effect of plateau pressure on day 3 on short-term mortality, including 11 studies and 22 study arms. Mean (SD) plateau pressure was 25.5 (3.4) $\mathrm{cm} \mathrm{H}_{2} \mathrm{O}$. (c) Meta-regression analysis of the effect of plateau pressure on day 7 on short-term mortality, including 12 studies and 24 study arms. Mean (SD) plateau pressure was $25.8(4.7) \mathrm{cm} \mathrm{H}_{2} \mathrm{O}$. SD: standard deviation.

remains until day 3 , and the effect tended to diminish by day 7. It is an important clinical question whether a lung protective strategy should be continued for a certain number of days. The results of this meta-analysis and meta-regression have made it possible to make a new suggestion regarding the duration of lung protective strategies.

When considering the effect of MV pressure on patient prognosis, it is important to recognize that MV pressure may correlate with the prognosis. In meta-analyses and clinical research reported so far $[8,9,36,37]$, the MV pressure, which is a continuous variable, was converted into a binary variable using a cut-off value to allow intergroup comparisons of its influence on outcomes. Within each group, the effects of different pressures on the outcomes were not uniform and may have had various degrees of correlation. Therefore, the effects of ventilator pressure on the prognosis could not be evaluated strictly from the results of previous clinical studies [8, 9]. To compensate for this weakness, we used a meta-regression method that considered the ventilator pressure as a continuous variable. The results on day 3 using the meta-regression analysis in Figure 2 show a slightly different tendency compared with the results obtained with the usual meta-analysis method, in which pressure is converted into a binary variable and analyzed. By analyzing these pressures as a binary variable, the result seemed to be either overestimated or underestimated.

\section{Implications for each stakeholder}

Accurate determination of the duration for which strict man- 

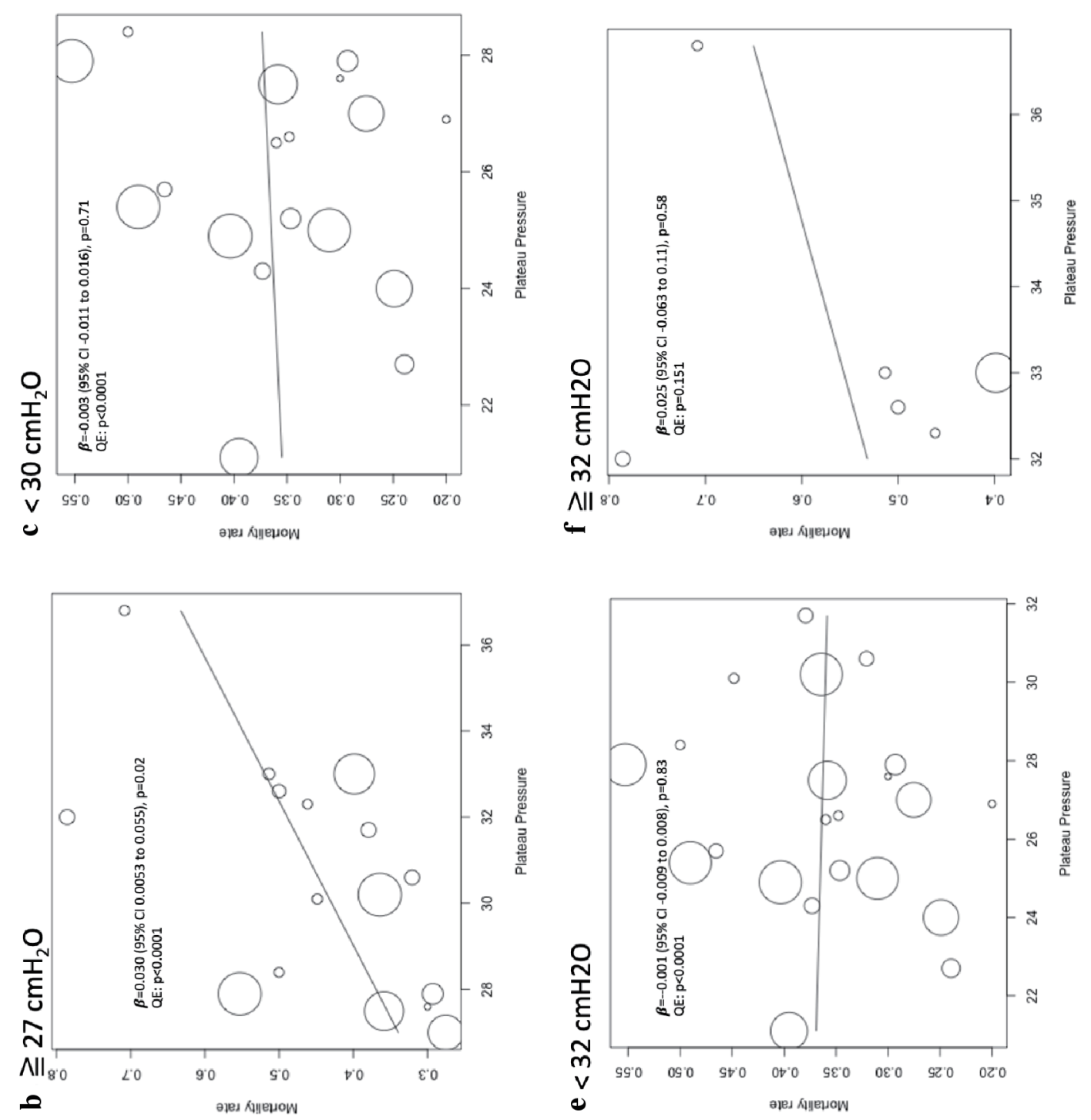

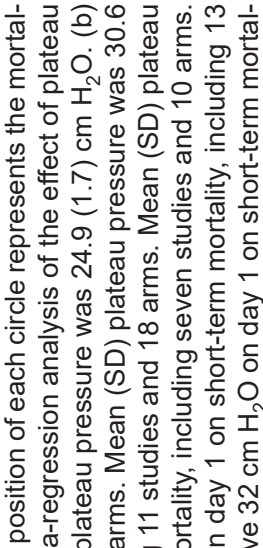

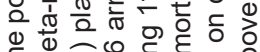

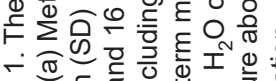

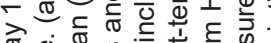
तิ

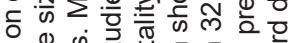

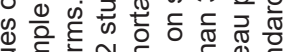

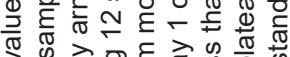
ᄂ

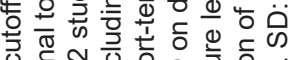

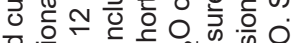

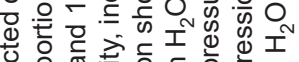

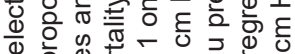
心

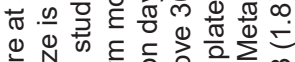
के ฏ)

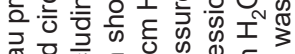

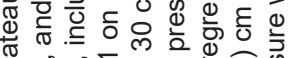
元 후

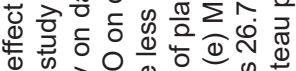

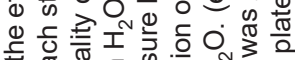
प⿺辶寸
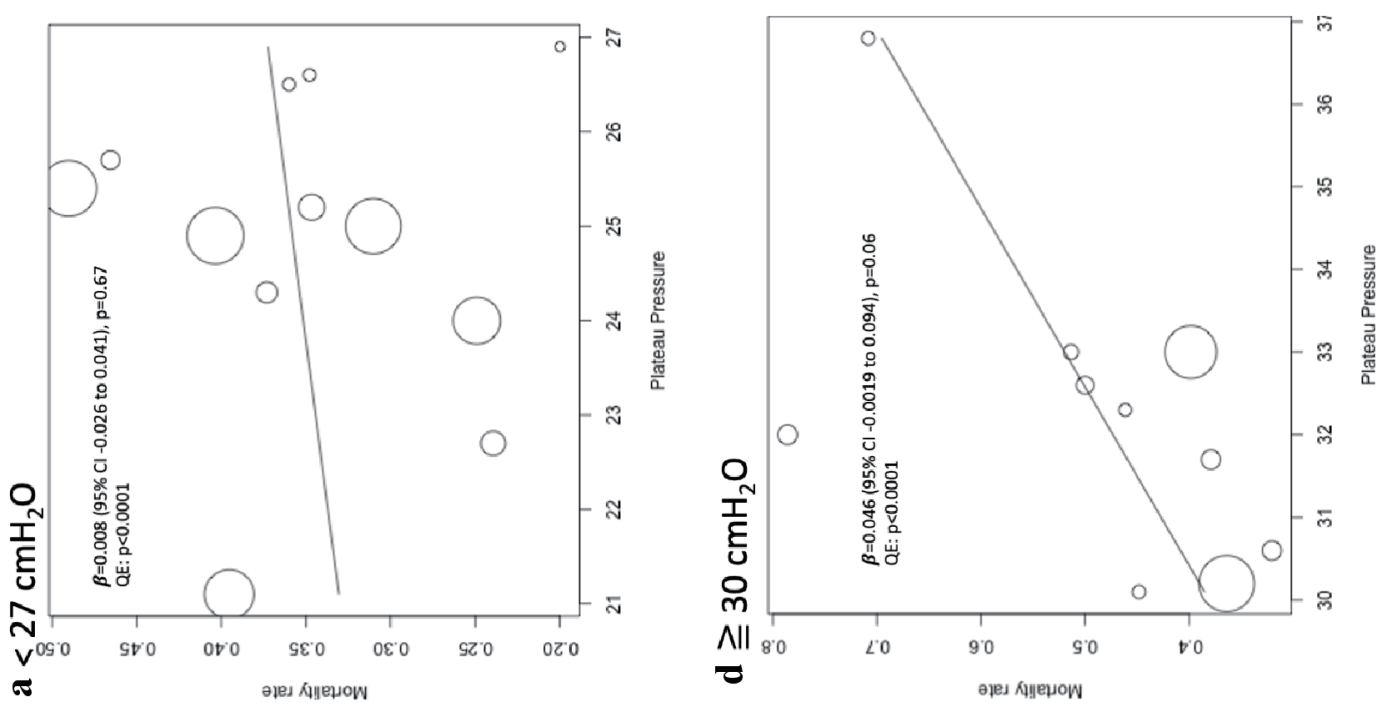

\% 든

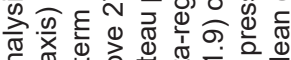
क 은

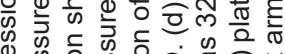
क人

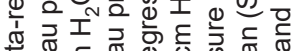

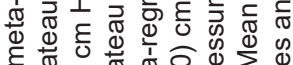

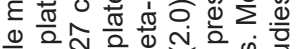

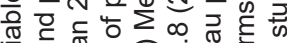

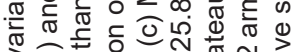

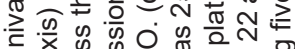
ป . mं

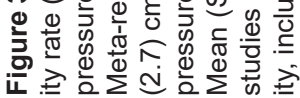



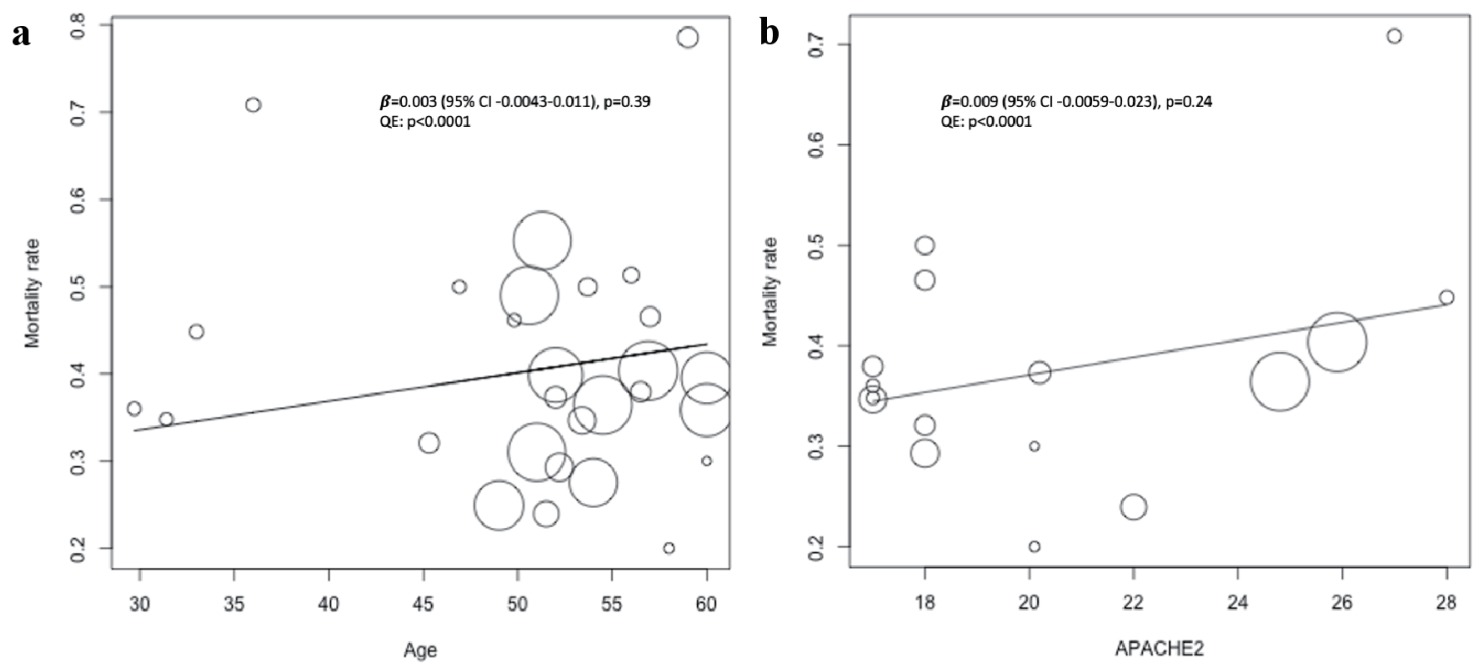

Figure 4. Univariable meta-regression of age and APACHE 2 scores at the time of admission to the ICU on short-term mortality. The position of each circle represents the mortality rate (y-axis) and plateau pressure (x-axis) of each study arm, and circle size is proportional to sample size. (a) Meta-regression of age on short-term mortality, including 14 studies and 28 arms. Mean (SD) age was 50.7 (8.5) years. (b) Meta-regression of APACHE 2 scores on short-term mortality, including eight studies and 16 arms. Mean (SD) APACHE 2 score was 20.5 (3.9). SD: standard deviation; APACHE: acute physiology and chronic health evaluation.

agement of MV pressure contributes to improvement in outcomes may facilitate MV management for physicians. If the tidal volume and the plateau pressure are to be limited below prespecified goals in the late phase of mechanical ventilation, a substantial number of patients may be difficult to reach the target tidal volume without sedation. For critical care physicians, the duration of the lung protection strategy is an essential, but unresolved question. Since the present study suggests that a lung protection strategy may not need to be followed in the later phase, clinicians may consider earlier discontinuation of sedatives, which may allow for earlier rehabilitation and, therefore, earlier liberation from mechanical ventilation.

\section{Limitations}

There are several limitations of this meta-analysis. First, the method used in this review is a meta-regression analysis, but it is only one type of meta-analysis, and a multivariate analysis was not performed by integrating individual data. This may have led to over- or under-estimation of the original results by summarizing the data for each individual participating in the study and then reintegrating it using the metaanalysis method. Second, the present meta-analysis included RCTs that compared plateau pressures but did not include RCTs that compared driving pressure or transpulmonary pressure. Recently, it was shown that driving pressure may have a greater influence on patient prognosis than plateau pressure [34]. However, a meta-analysis with a limited number of RCTs that compared the driving pressure has limited value. Although a meta-analysis of RCTs $[38,39]$ that evaluate transpulmonary pressure is worthy of consideration, a comparison of plateau pressure was not performed in these studies, but the superiority of a personalized ventilation strat- egy using transpulmonary pressure was assessed. Also, the frequency of esophageal pressure measurements reported in the Lung Safe Study [35] was 1.2\%, suggesting that ventilation strategy using transpulmonary pressure is not yet standard management. Third, the meta-regression analysis used in this review is different from standard meta-regression that investigates whether the difference in study characteristics (e.g., age, time, and other such factors) may account for the heterogeneity of effect size of each included study. The method of meta-regression analysis used in this review considered each of the two study arms in RCTs as an individual group, and the mortality ratio in each group, not effect size, was used as the dependent variable. The mortality ratio in each group was then evaluated by regression analysis. Although a similar technique may be used for meta-regression analysis of certain studies [40], the study results should be interpreted with caution. Finally, in this meta-analysis, univariable metaregression analysis was conducted, not multivariable metaregression analysis because the possible confounding factors, such as etiology of ARDS, could not be collected from the data in each study. There may be several confounding factors affecting mortality and plateau pressure, such as etiology of ARDS and transpulmonary pressure, which were not considered in this meta-analysis, so the interpretation of the results should not be exaggerated.

\section{Conclusions}

This review suggests that MV management, restricting plateau pressure, for at least several days after hospitalization may be critical for improving prognosis. According to this meta-analysis, the optimal cut-off values for plateau pressure are around $27 \mathrm{~cm} \mathrm{H}_{2} \mathrm{O}$. However, since this is a meta-analysis with recognized limitations, it is necessary to conduct further 
studies, including individual meta-analyses and large-scale interventions.

\section{Supplementary Material}

Suppl 1. Search strategy.

Suppl 2. Risk of bias table on short-term mortality.

Suppl 3. Forest plots of short-term mortality according to the plateau pressure on day 1 at selected cut-off values.

Suppl 4. Forest plots of short-term mortality according to plateau pressure on day 3 at selected cut-off values.

Suppl 5. Forest plots of short-term mortality according to plateau pressure on day 7 at selected cut-off values.

\section{Acknowledgments}

The authors would like to thank all study participants, and two authors who provided the data of their meta-analyses.

\section{Financial Disclosure}

This research did not receive any specific grant from funding agencies in the public, commercial, or not-for-profit sectors.

\section{Conflict of Interest}

None to declare.

\section{Informed Consent}

Not applicable.

\section{Author Contributions}

HY contributed to the study concept and design, screening for relevant studies, and drafting of the manuscript. TN and TK contributed to the study concept and design, and screening for relevant studies. MS contributed to the study concept and design, and critical revision of the manuscript for important intellectual content. AKL contributed to critical revision of the manuscript for important intellectual content. EN supervised the process of systematic review. TA and RR supervised the process of analysis as a biostatistician. TT, AKL, and SH were consultants on clinically relevant issues.

\section{Data Availability}

The authors declare that data supporting the findings of this study are available within the article.

\section{Abbreviations}

ALI: acute lung injury; ARDS: acute respiratory distress syndrome; CENTRAL: Cochrane Central Register of Controlled Trials; CI: confidence intervals; HFOV: high-frequency oscillation ventilation; $\mathrm{MV}$ : mechanical ventilation; PEEP: positive end-expiratory pressure; PRISMA: Preferred Reporting Items for Systematic review and Meta-Analyses; RCT: randomized controlled trial; RevMan: Review Manager; RR: relative risk; VILI: ventilator-induced lung injury

\section{References}

1. Writing Group for the Alveolar Recruitment for Acute Respiratory Distress Syndrome Trial Investigators, Cavalcanti AB, Suzumura EA, Laranjeira LN, Paisani DM, Damiani LP, Guimaraes HP, et al. Effect of lung recruitment and titrated positive end-expiratory pressure (PEEP) vs low PEEP on mortality in patients with acute respiratory distress syndrome: a randomized clinical trial. JAMA. 2017;318(14):1335-1345.

2. Zhou Y, Jin X, Lv Y, Wang P, Yang Y, Liang G, Wang B, et al. Early application of airway pressure release ventilation may reduce the duration of mechanical ventilation in acute respiratory distress syndrome. Intensive Care Med. 2017;43(11):1648-1659.

3. Meade MO, Cook DJ, Guyatt GH, Slutsky AS, Arabi YM, Cooper DJ, Davies AR, et al. Ventilation strategy using low tidal volumes, recruitment maneuvers, and high positive end-expiratory pressure for acute lung injury and acute respiratory distress syndrome: a randomized controlled trial. JAMA. 2008;299(6):637-645.

4. Kacmarek RM, Villar J, Sulemanji D, Montiel R, Ferrando C, Blanco J, Koh Y, et al. Open lung approach for the acute respiratory distress syndrome: a pilot, randomized controlled trial. Crit Care Med. 2016;44(1):32-42.

5. Fish E, Novack V, Banner-Goodspeed VM, Sarge T, Loring S, Talmor D. The Esophageal Pressure-Guided Ventilation 2 (EPVent2) trial protocol: a multicentre, randomised clinical trial of mechanical ventilation guided by transpulmonary pressure. BMJ Open. 2014;4(9):e006356.

6. Talmor D, Sarge T, Malhotra A, O'Donnell CR, Ritz $\mathrm{R}$, Lisbon A, Novack $\mathrm{V}$, et al. Mechanical ventilation guided by esophageal pressure in acute lung injury. N Engl J Med. 2008;359(20):2095-2104.

7. Villar J, Ambros A, Soler JA, Martinez D, Ferrando C, Solano R, Mosteiro F, et al. Age, $\mathrm{PaO} 2 / \mathrm{FIO} 2$, and plateau pressure score: a proposal for a simple outcome score in patients with the acute respiratory distress syndrome. Crit Care Med. 2016;44(7):1361-1369.

8. Acute Respiratory Distress Syndrome Network, Brower RG, Matthay MA, Morris A, Schoenfeld D, Thompson BT, Wheeler A. Ventilation with lower tidal volumes as compared with traditional tidal volumes for acute lung injury and the acute respiratory distress syndrome. N Engl J Med. 2000;342(18):1301-1308.

9. Petrucci N, De Feo C. Lung protective ventilation strat- 
egy for the acute respiratory distress syndrome. Cochrane Database Syst Rev. 2013;2:CD003844.

10. Collaboration TC. RevMan 5 Download and Installion. Secondary RevMan 5 Download and Installion. https:// community.cochrane.org/help/tools-and-software/revman-5/revman-5-download/installation. 2014. [Accessed September 29, 2019].

11. Laffey JG, Bellani G, Pham T, Fan E, Madotto F, Bajwa EK, Brochard L, et al. Potentially modifiable factors contributing to outcome from acute respiratory distress syndrome: the LUNG SAFE study. Intensive Care Med. 2016;42(12):1865-1876.

12. Shamseer L, Moher D, Clarke M, Ghersi D, Liberati A, Petticrew M, Shekelle P, et al. Preferred reporting items for systematic review and meta-analysis protocols (PRISMA-P) 2015: elaboration and explanation. BMJ. 2015;350:g7647.

13. Yasuda H, Nishimura T, Kamo T, Sanui M, Nango E, Abe $\mathrm{T}$, Takebayashi $\mathrm{T}$, et al. Optimal plateau pressure for patients with acute respiratory distress syndrome: a protocol for a systematic review and meta-analysis with meta-regression. BMJ Open. 2017;7(5):e015091.

14. Ards Definition Task Force, Ranieri VM, Rubenfeld GD, Thompson BT, Ferguson ND, Caldwell E, Fan E, et al. Acute respiratory distress syndrome: the Berlin Definition. JAMA. 2012;307(23):2526-2533.

15. Bernard GR, Artigas A, Brigham KL, Carlet J, Falke K, Hudson L, Lamy M, et al. The American-European Consensus Conference on ARDS. Definitions, mechanisms, relevant outcomes, and clinical trial coordination. Am J Respir Crit Care Med. 1994;149(3 Pt 1):818-824.

16. Collaboration. TC. Data Collection Form-Intervention review for RCTs only. Secondary Data Collection FormIntervention review for RCTs only. https://dplp.cochrane. org/data-extraction-forms. 2014. [Accessed September 29, 2019].

17. Collaboration. TC. Cochrane Handbook for Systematic Reviews of Interventions. https://training.cochrane.org/ handbook. 2011. [Accessed September 29, 2019].

18. Collaboration. TC. Table 8.5.d: Criteria for judging risk of bias in the 'Risk of bias' assessment tool. Secondary Table 8.5.d: Criteria for judging risk of bias in the 'Risk of bias' assessment tool. http://handbook-5-1.cochrane.org. 2011. [Accessed September 29, 2019].

19. Higgins JP, Thompson SG. Quantifying heterogeneity in a meta-analysis. Stat Med. 2002;21(11):1539-1558.

20. Sterne JA, Sutton AJ, Ioannidis JP, Terrin N, Jones DR, Lau J, Carpenter J, et al. Recommendations for examining and interpreting funnel plot asymmetry in meta-analyses of randomised controlled trials. BMJ. 2011;343:d4002.

21. Brower RG, Shanholtz CB, Fessler HE, Shade DM, White P, Jr., Wiener CM, Teeter JG, et al. Prospective, randomized, controlled clinical trial comparing traditional versus reduced tidal volume ventilation in acute respiratory distress syndrome patients. Crit Care Med. 1999;27(8):1492-1498.

22. Villar J, Kacmarek RM, Perez-Mendez L, Aguirre-Jaime A. A high positive end-expiratory pressure, low tidal volume ventilatory strategy improves outcome in persistent acute respiratory distress syndrome: a randomized, controlled trial. Crit Care Med. 2006;34(5):1311-1318.

23. Hodgson CL, Tuxen DV, Davies AR, Bailey MJ, Higgins AM, Holland AE, Keating JL, et al. A randomised controlled trial of an open lung strategy with staircase recruitment, titrated PEEP and targeted low airway pressures in patients with acute respiratory distress syndrome. Crit Care. 2011;15(3):R133.

24. Agarwal R, Srinivasan A, Aggarwal AN, Gupta D. Adaptive support ventilation for complete ventilatory support in acute respiratory distress syndrome: a pilot, randomized controlled trial. Respirology. 2013;18(7):1108-1115.

25. Amato MB, Barbas CS, Medeiros DM, Magaldi RB, Schettino GP, Lorenzi-Filho G, Kairalla RA, et al. Effect of a protective-ventilation strategy on mortality in the acute respiratory distress syndrome. N Engl J Med. 1998;338(6):347-354.

26. Brower RG, Lanken PN, MacIntyre N, Matthay MA, Morris A, Ancukiewicz M, Schoenfeld D, et al. Higher versus lower positive end-expiratory pressures in patients with the acute respiratory distress syndrome. N Engl J Med. 2004;351(4):327-336.

27. Mercat A, Richard JC, Vielle B, Jaber S, Osman D, Diehl JL, Lefrant JY, et al. Positive end-expiratory pressure setting in adults with acute lung injury and acute respiratory distress syndrome: a randomized controlled trial. JAMA. 2008;299(6):646-655.

28. Esteban A, Alia I, Gordo F, de Pablo R, Suarez J, Gonzalez G, Blanco J. Prospective randomized trial comparing pressure-controlled ventilation and volume-controlled ventilation in ARDS. For the Spanish Lung Failure Collaborative Group. Chest. 2000;117(6):1690-1696.

29. Brochard L, Roudot-Thoraval F, Roupie E, Delclaux C, Chastre J, Fernandez-Mondejar E, Clementi E, et al. Tidal volume reduction for prevention of ventilator-induced lung injury in acute respiratory distress syndrome. The Multicenter Trail Group on Tidal Volume reduction in ARDS. Am J Respir Crit Care Med. 1998;158(6):18311838.

30. Claesson J, Freundlich M, Gunnarsson I, Laake JH, Vandvik PO, Varpula T, Aasmundstad TA, et al. Scandinavian clinical practice guideline on mechanical ventilation in adults with the acute respiratory distress syndrome. Acta Anaesthesiol Scand. 2015;59(3):286-297.

31. Hashimoto S, Sanui M, Egi M, Ohshimo S, Shiotsuka J, Seo R, Tanaka R, et al. The clinical practice guideline for the management of ARDS in Japan. J Intensive Care. 2017;5:50.

32. Rhodes A, Evans LE, Alhazzani W, Levy MM, Antonelli M, Ferrer R, Kumar A, et al. Surviving sepsis campaign: international guidelines for management of sepsis and septic shock: 2016. Intensive Care Med. 2017;43(3):304377.

33. Nishida O, Ogura H, Egi M, Fujishima S, Hayashi Y, Iba $\mathrm{T}$, Imaizumi H, et al. The Japanese clinical practice guidelines for management of sepsis and septic shock 2016 (JSSCG 2016). J Intensive Care. 2018;6:7.

34. Amato MB, Meade MO, Slutsky AS, Brochard L, Costa EL, Schoenfeld DA, Stewart TE, et al. Driving pressure 
and survival in the acute respiratory distress syndrome. N Engl J Med. 2015;372(8):747-755.

35. Bellani G, Laffey JG, Pham T, Fan E, Brochard L, Esteban A, Gattinoni L, et al. Epidemiology, Patterns of Care, and Mortality for Patients With Acute Respiratory Distress Syndrome in Intensive Care Units in 50 Countries. JAMA. 2016;315(8):788-800.

36. Fuller BM, Mohr NM, Drewry AM, Carpenter CR. Lower tidal volume at initiation of mechanical ventilation may reduce progression to acute respiratory distress syndrome: a systematic review. Crit Care. 2013;17(1):R11.

37. Burns KE, Adhikari NK, Slutsky AS, Guyatt GH, Villar J, Zhang H, Zhou Q, et al. Pressure and volume limited ventilation for the ventilatory management of patients with acute lung injury: a systematic review and meta-analysis. PLoS One. 2011;6(1):e14623.
38. Li J, Luo Z, Li X, Huang Z, Han J, Li Z, Zhou Z, et al. [Effect of different transpulmonary pressures guided mechanical ventilation on respiratory and hemodynamics of patients with ARDS: a prospective randomized controlled trial]. Zhonghua Wei Zhong Bing Ji Jiu Yi Xue. 2017;29(1):39-44.

39. Beitler JR, Sarge T, Banner-Goodspeed VM, Gong MN, Cook D, Novack V, Loring SH, et al. Effect of titrating positive end-expiratory pressure (PEEP) with an esophageal pressure-guided strategy vs an empirical high PEEP-Fio2 strategy on death and days free from mechanical ventilation among patients with acute respiratory distress syndrome: a randomized clinical trial. JAMA. 2019;321(9):846-857.

40. Viechtbauer. Conducting meta-analyses in $\mathrm{R}$ with the metafor package. J Stat Softw 2010;36. 